\title{
Reentrant cavities as electromechanical transducers
}

\author{
J. J. Barroso, a) P. J. Castro, O. D. Aguiar, and L. A. Carneiro \\ National Institute for Space Research/INPE, S. José dos Campos 12227-010, São Paulo, Brazil
}

(Received 11 September 2003; accepted 25 January 2004; published 15 March 2004)

The present work quantifies the dependence of the tunable frequency range on the gap spacing between the end of the conical post and the cavity top plate in reentrant $1.0 \mathrm{GHz}$ klystron cavities. Fabricated from aluminum, the cavities tested are $80 \mathrm{~mm}$ in diameter with a top plate $1 \mathrm{~mm}$ thick. Experiments performed on such cavities have shown tuning coefficients (change in resonant frequency due to variation of the capacitive gap) as high as $40.0 \mathrm{MHz} / \mu \mathrm{m}$, thereby demonstrating the capability of reentrant cavities as electromechanical transducers in resonant mass gravitational wave antennas. For cavity-based transducers ten times as small as the cavities tested here, this result translates into a tuning coefficient 100 times higher. (c) 2004 American Institute of Physics.

[DOI: $10.1063 / 1.1688438$ ]

\section{INTRODUCTION}

Strong electric fields for accelerating or modulating an electron beam find important microwave applications in linear accelerators and rf power sources, where for a cavity with a given stored energy the strongest field is desired. ${ }^{1,2} \mathrm{Rf}$ cavity technology has developed significantly in the last decade with the introduction of improved surface treatment and assembly procedures, advanced materials, and by a better understanding of the phenomena which have in the past limited the performance of superconducting cavities, such as, e.g., multipacting, field emission, and quenches. ${ }^{3}$ Accelerating gradients in excess of $50 \mathrm{MV} / \mathrm{m}$ and electrical $Q$ factors of about $10^{10}$ are no longer out of reach. ${ }^{4}$ Several simulation computer codes-for example, SUPERFISH, ${ }^{2}$ HFSS,${ }^{5}$ MAFIA, ${ }^{5}$ or $\mathrm{CST}^{6}$ - are available to solve the electromagnetic field equations for typical cavity geometries. Of particular interest in those solutions are the peak surface electric and magnetic fields, the shunt impedance (which determines the cavity losses at a given surface resistance and gradient), and the stored energy. Concerning mechanical stability, an operational parameter especially important in pulsed operation is the Lorentz force detuning coefficient, which influences the choice of the material thickness and has to be taken into account in the mechanical design of the cavity. ${ }^{3}$ In the design stage, the criterion is to maximize the shunt impedance while obtaining intense electric fields uniformly distributed on the cavity irises. Also relying on intense fields to increase the energy sensitivity, electromagnetic cavity-based transducers ${ }^{7}$ operate at high fields to maximize the electrical coupling to an external mechanical transformer. A cavity of this sort is usually accomplished by a reentrant klystron cavity (Fig. 1) where intense electric fields develop across a short gap.

In this article, we examine both theoretically and experimentally the resonance properties of azimuthally symmetric reentrant cavities. Such components are used as parametric transducers to continually monitor the vibrational state of

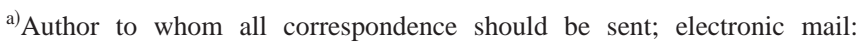
barroso@plasma.inpe.br
}

mass gravitational wave antennas through modulation of the capacitive gap. ${ }^{7-9}$ Here we investigate the relationship between the resonant frequency and the cavity dimensions with emphasis on how the frequency varies when flexing the top plate by means of a directed force.

\section{CAVITY ANALYSIS}

As pictured in Fig. 1, for a small gap spacing the electric field lines of the corresponding operation mode run in the gap region as from one plate to the other of a parallel plate capacitor, whereas in the rest of the cavity the field is substantially as in a coaxial line. On condition that the gap spacing $d$ is much shorter than the resonant wavelength the concept of lumped circuit elements becomes meaningful, whereby we treat the reentrant cavity as a shorted coaxial line terminated by a capacitor (Fig. 2). Thus for a line of length $l$, outer diameter $2 r_{2}$, inner diameter $2 r_{1}$, and terminal capacitance $C$ the resonance condition requires that the loop impedance be zero, so that

$$
j Z_{0} \tan \beta_{0} l+\frac{1}{j \omega_{0} C}=0
$$

where $\quad \beta_{0}=2 \pi / \lambda_{0}=\omega_{0} \sqrt{\mu_{0} \varepsilon_{0}}, \quad Z_{0}=(1 / 2) \sqrt{\mu_{0} \varepsilon_{0}} \ln \left(r_{2} r_{1}\right)$, and in a first approximation the gap capacitance is expressed as $C=\varepsilon_{0}\left(\pi r_{1}^{2} / d\right)$. Assuming $\beta_{0} l \ll 1$, Eq. (1) simplifies to $\omega_{0} C Z_{0} \beta_{0} l=1$ giving the resonant wavelength

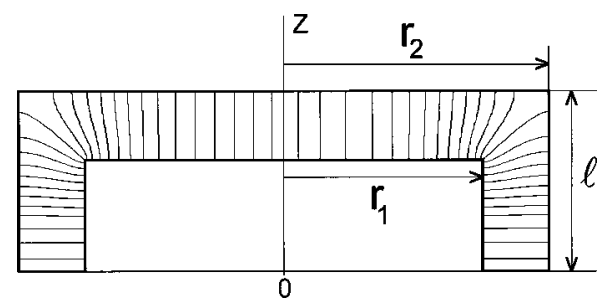

FIG. 1. Reentrant cavity schematic showing electric field lines. 


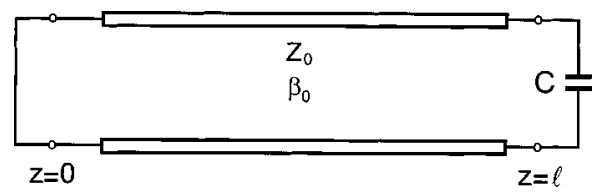

FIG. 2. Approximate equivalent circuit of the cavity in Fig. 1.

$$
\lambda_{0}=r_{1} \sqrt{2 \pi^{2} \frac{l}{d} \ln \frac{r_{2}}{r_{1}}} .
$$

We note that if $d$ is small compared to $l$, as we are assuming, then $\lambda_{0}$ is large compared to $r_{1}$. If, as is usually the case $r_{1}$ is of the same order of magnitude as $r_{2}$ and $l$, then this means that $\lambda_{0}$ is large compared to all the dimensions of the cavity, justifying our assumption that the cavity can be treated as a lumped constant problem. Then if we wish to design a cavity for a given $\lambda_{0}$, we see that the smaller is $d$, the smaller the cavity dimensions become, so that we can make in this way a conveniently small cavity resonant at a long wavelength. Although enlightening, the simple formula (2) does not provide an accurate estimate of the resonant frequency (in some cases the error may be larger than $40 \%$ ) as its derivation lacks the cavity capacitance that accounts for the fringing fields in the transition region intermediate the coaxial and gap spaces. Calculated as ${ }^{10}$

$$
C_{1}=4 \varepsilon_{0} r_{1} \ln \frac{e \sqrt{\left(r_{2}^{2}-r_{1}^{2}\right)+l^{2}}}{2 d},
$$

the cavity capacitance $C_{1}$ when added to $C_{0}$ much improves

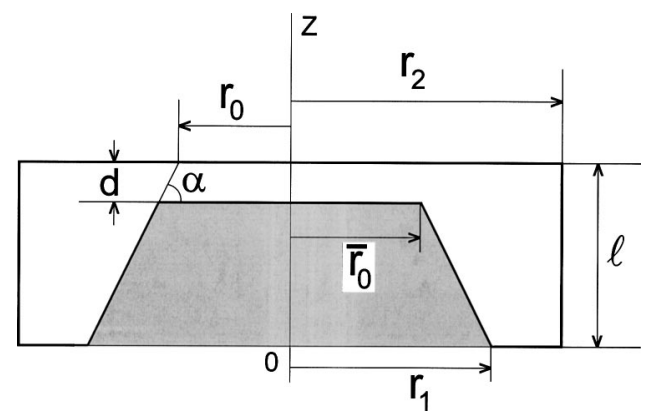

FIG. 3. Definition of geometrical parameters for the reentrant cavity with coaxial conical insert.

the accuracy of the equivalent circuit. Generalizing the configuration shown in Fig. 1, a reentrant cavity with a coaxial conical insert (Fig. 3) has been modeled by Fujisawa ${ }^{10}$ as a lumped $L C$ circuit leading to the following parameters:

$$
\begin{aligned}
\frac{L}{\mu_{0}}= & \frac{l}{2 \pi}\left(\ln \frac{e r_{2}}{r_{1}}-\frac{r_{0}}{r_{1}-r_{0}} \ln \frac{r_{1}}{r_{0}}\right), \\
\frac{C_{0}}{\varepsilon_{0}}= & \frac{\pi \bar{r}_{0}^{2}}{d}, \\
\frac{C_{1}}{\varepsilon_{0}}= & \frac{\pi\left(r_{0}^{2}-\bar{r}_{0}^{2}\right)}{d}+\frac{2 \pi}{\alpha} \\
& \times\left(r_{0} \ln \frac{e l_{M} \sin \alpha}{d}+\frac{d \cot \alpha}{2} \ln \frac{\sqrt{e} l_{M} \sin \alpha}{d}\right),
\end{aligned}
$$

where $e$ is the exponential constant $(e=2.71828, \ldots$,$) , and$

$$
l_{M}=\frac{\sqrt{\left[2\left(r_{1}-r_{0}\right)^{2}+3\left(r_{2}-r_{1}\right)\left(r_{1}+r_{2}-2 r_{0}\right)\right]^{2}+l^{2}\left(3 r_{2}-2 r_{1}-r_{0}\right)^{2}}}{3\left(2 r_{2}-r_{1}-r_{0}\right)}
$$

$$
\alpha=\tan ^{-1} \frac{l-d}{r_{1}-\bar{r}_{0}},
$$

for which the error incurred in estimating the resonant frequency $f_{0}=1 / 2 \pi \sqrt{L\left(C_{0}+C_{1}\right)}$ lies within a few percent as has been verified by Fujisawa ${ }^{10}$ upon comparison with experiments. Accordingly, the accuracy of the formulas becomes better for larger $r_{0} / l_{M}$ and smaller $l_{M} / \lambda_{0}$, respectively, the post radius and the resonant wavelength compared with the relative size of the cavity.

On the basis of relations (4)-(8), we examine below how the electrodynamical properties of the reentrant cavity relate to the shape of the coaxial insert by considering two types of posts: a truncated cone and a circular cylinder, the latter of which the general expressions (6)-(8) apply when $\alpha=\pi / 2$ $\left(r_{1}=r_{0}\right.$, Fig. 3). Markedly different for each coaxial insert, the plots in Fig. 4 show the dependence of resonant frequency $f_{0}$ on radius $r_{1}$ for fixed major radius $r_{2}$ $(=40.0 \mathrm{~mm})$ and cavity length $l(=20.0 \mathrm{~mm})$ with gap spacing $d$ varying from 0.1 to $0.5 \mathrm{~mm}$ in steps of $0.1 \mathrm{~mm}$. For the circularly cylindrical insert [Fig. 4(a)], $f_{0}$ starts decreasing for increasing $r_{1}$ and after reaching a flat region all the curves come nearer to each other at large values of $r_{1}$, eventually merging into a single curve in which the particular behavior entailed separately by the gap $d$ on each curve is lost. With most of the electromagnetic energy stored in the gap region and with the electric-field lines running axially, this regime $\left(r_{1} \rightarrow r_{2}\right)$ closely resembles the $\mathrm{TM}_{010}$-mode operation in a circular cavity. In fact we note that the frequency curves going upward tend to an asymptotic value that is consistent with the resonant frequency of a $\mathrm{TM}_{010}$-mode cavity with radius $r_{2}=40.0 \mathrm{~mm}$, i.e., $f_{\mathrm{TM} 010}=(15 / \pi)\left(\chi_{01} / r_{2}\right)$ $=2.87 \mathrm{GHz}$, [where $\chi_{01}=2.4048$ is the first zero of the Bessel function $\left.J_{0}(\chi)\right]$. By contrast, for the cavity with the conical insert [Fig. 4(b)] all the frequency curves slope upward and run parallel to each other as $r_{1}$ increases. Moreover, we remark that the frequency separation given by the upper and innermost curves, for instance, at $r_{1}=2.0 \mathrm{~cm}$, for the cavity with uniform post $(0.57 \mathrm{GHz})$ is nearly half that for the cavity with tapered insert $(0.97 \mathrm{GHz})$, which thus exhibits higher sensitivity to variations in $d$.

To see the effect of the top radius of the post on the 


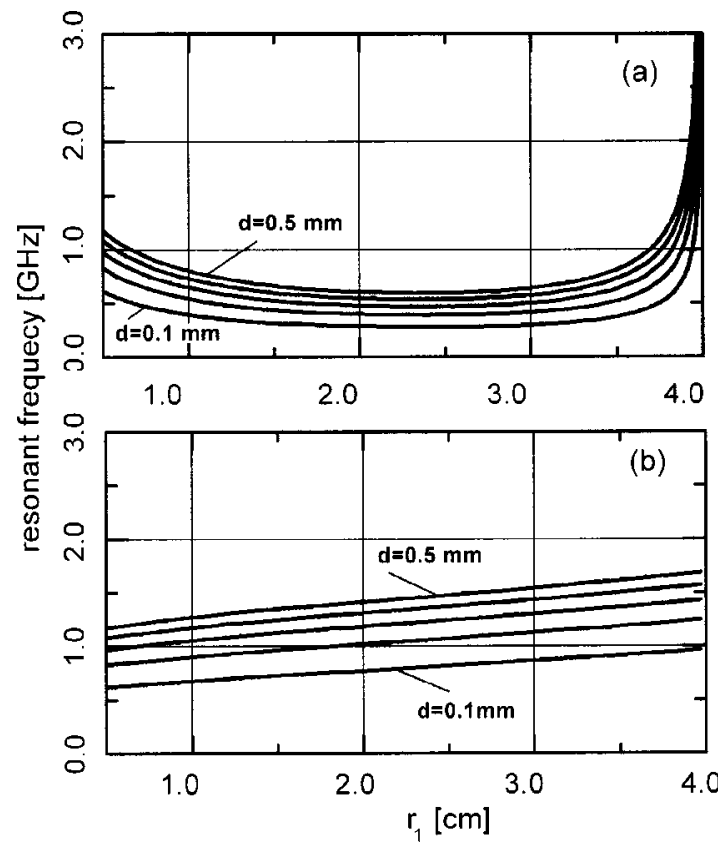

FIG. 4. Resonant frequency as a function of inner radius $r_{1}$ for reentrant cavities with (a) circularly cylindrical and (b) conical inserts. The curves are parametrized at increments of $0.1 \mathrm{~mm}$ in the gap $d$. In both figures, $r_{2}$ $=40.0 \mathrm{~mm}$ and $l=20.0 \mathrm{~mm}$.

resonant frequency, in Fig. 5 we show $f_{0}$ as a function of the gap $d$ in cavities having $\bar{r}_{0}=3.0 \mathrm{~mm}, \bar{r}_{0}=10.0 \mathrm{~mm}$ with the inner radius ranging from $r_{1}=10.0 \mathrm{~mm}$ (lower curve) to $r_{1}$ $=20.0 \mathrm{~mm}$ (upper curve), at a $5.0 \mathrm{~mm}$ increment. The innermost curve with $\bar{r}_{0}=r_{1}=10.0 \mathrm{~mm}$, corresponding to a cylinder, indicates that cylindrical posts impart lower frequency sensitivity to variation in the gap $d$ when compared at the same top radius $\bar{r}_{0}$ with conical posts. Besides increasing with $r_{1}$ for fixed $d$, we see that $f_{0}$ greatly increases as $\bar{r}_{0}$ decreases. Then we expect that smaller $\bar{r}_{0}$ and larger $r_{1}$ lead to higher tuning coefficients.

\section{EXPERIMENT}

The resonance properties of a reentrant cavity with conical insert is experimentally examined by looking at the effect on the resonant frequency of reducing the gap spacing through the application of a bending force at the center of the

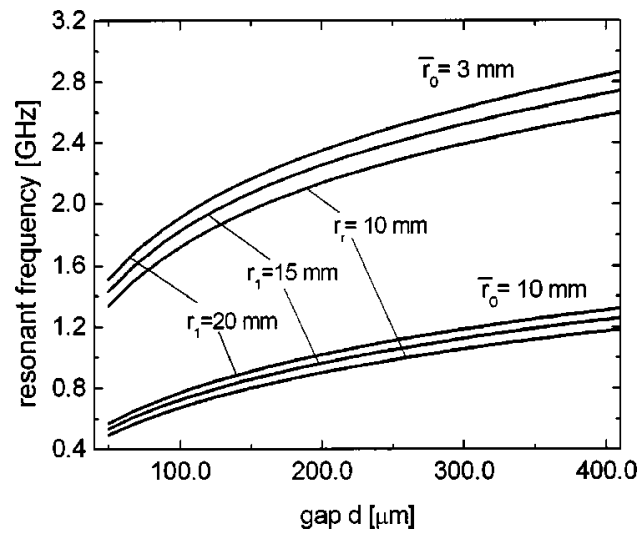

FIG. 5. Gap spacing dependence of resonant frequency at fixed values of base and top radii $r_{1}$ and $\bar{r}_{0}$.

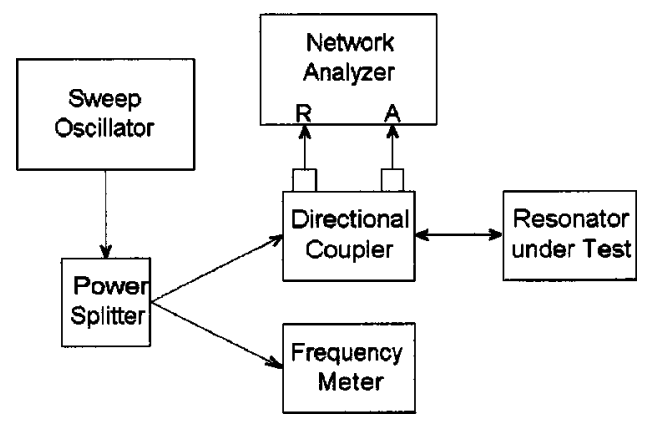

FIG. 6. Experimental setup to measure resonant frequencies.

circular top plate with clamped edges. Fabricated from aluminum, the cavity has dimensions to allow operation in the klystron mode (Fig. 1) around $1.0 \mathrm{GHz}$, a value well below the cutoff frequencies of potentially competing modes, since the major radius $r_{2}(=40.0 \mathrm{~mm})$ being constrained to $r_{2}$ $<\lambda_{0} \chi_{11} /(2 \pi)$ where $\chi_{11}=1.8411$, the first root of $J_{1}^{\prime}(\chi)$ $=0$, sets a cutoff frequency $f_{c}=(c / 2 \pi)\left(\chi_{11} / r_{2}\right)=2.5 \mathrm{GHz}$ for propagation of either TM or TE modes.

Resonant frequencies have been measured by using the reflection-type circuit configuration in Fig. 6 where the cavity fields are both excited and detected by means of a single electric probe inserted through a 1.0-mm-diam hole drilled halfway across the cylindrical wall, as pictured in Fig. 7. Five coaxial inserts (with dimensions given in Table I) have been tested on a circular cavity of radius $r_{2}=40.00 \mathrm{~mm}$ and height $l=20.0 \mathrm{~mm}$.

On applying a deflection force (using a set of calibrated weights) we then measured the corresponding downshifted frequencies, which are plotted in Fig. 8. Comparing inserts 3 and 4 , we see that the corresponding tuning curves are somewhat similar, differing by a frequency shift controlled by the gap spacing $d$. A comparison of insert 3 with insert 5 shows that the larger $\bar{r}_{0}$ of insert 3 leaves its tuning curve broader. Moreover, insert 1 having the larger $r_{1}$ and the smaller $\bar{r}_{0}$

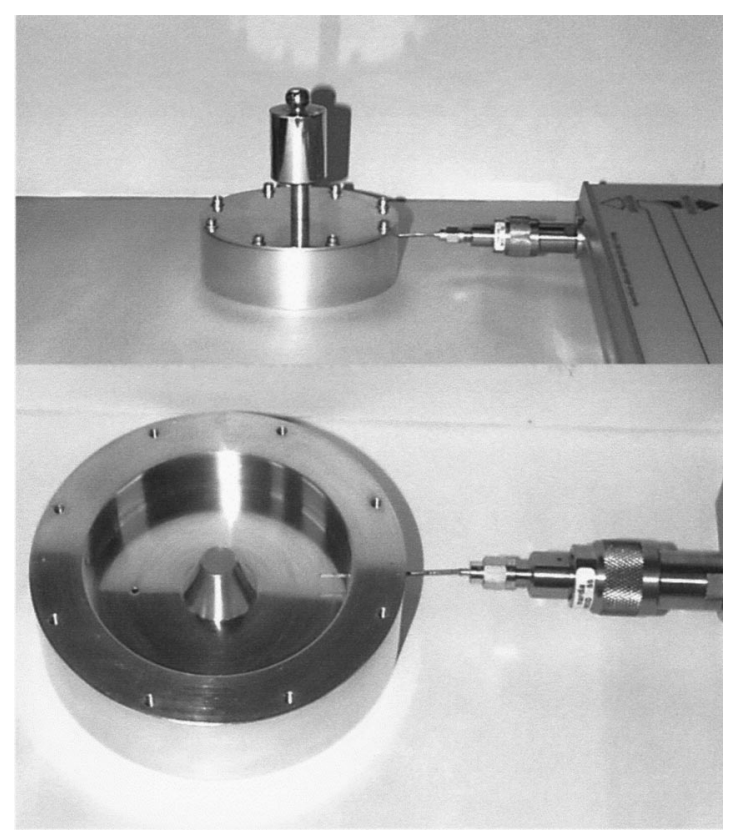

FIG. 7. Reentrant cavity under test. 
TABLE I. Gap spacing $(d)$, bottom $\left(r_{1}\right)$, and top $\left(\bar{r}_{0}\right)$ radii of the inserts tested.

\begin{tabular}{cccc}
\hline \hline Insert number & $d / \mathrm{mm}$ & $r_{1} / \mathrm{mm}$ & $\bar{r}_{0} / \mathrm{mm}$ \\
\hline 1 & 0.10 & 20.0 & 1.5 \\
2 & 0.10 & 10.0 & 1.5 \\
3 & 0.30 & 20.0 & 5.0 \\
4 & 0.40 & 20.0 & 5.0 \\
5 & 0.30 & 20.0 & 3.0 \\
\hline \hline
\end{tabular}

produces the steepest curve. Insert 2 , with a smaller $r_{1}$, has the effect of flattening the curve relative to curve 1 . So the greatest sensitivity of insert 1 is conferred by its large $r_{1}$ in conjunction with a small $\bar{r}_{0}$. Of course the surface area $\pi \bar{r}_{0}^{2}$ should not be taken infinitely small otherwise the capacitance effect would disappear; indeed, the optimum value of $\bar{r}_{0}=1.5 \mathrm{~mm}$ has been found for the range $5.0 \mathrm{~mm} \leqslant r_{1}$ $\leqslant 30.0 \mathrm{~mm}$.

To quantitatively assess the sensitivity rendered by insert 1, we plot in Fig. 9 the corresponding measured frequencies and calculated derivatives against the variation of the capacitive gap $x=d_{0}-\delta_{\max }$, where $d_{0}=0.10 \mathrm{~mm}$ denotes the nominal gap spacing and $\delta_{\max }$ is the maximal deflection produced by an external force acting on the top plate (Fig. 10). The deformation of the plate is determined from the following expression that gives the deflection due to pure bending of a clamped circular plate loaded at the center.: ${ }^{11}$

$$
\delta(r, P)=\frac{\operatorname{Pr}^{2}}{8 \pi D} \ln \frac{r}{r_{2}}+\frac{P}{16 \pi D}\left(r_{2}^{2}-r^{2}\right),
$$

where $P$ is the load applied, $D=E h^{3} / 12\left(1-\nu^{2}\right)$ denotes the flexural rigidity of the plate of thickness $h=1.0 \mathrm{~mm}$, modulus of elasticity $E=69.0 \mathrm{GPa}$, and Poisson's ratio $\nu=0.3$.

In Fig. 9, the displacement sensitivity $d f / d x$ rapidly increases as the gap narrows, reaching the sensitivity of 40.0 $\mathrm{MHz} / \mu \mathrm{m}$ at $x \simeq 50.0 \mu \mathrm{m}$ which corresponds to a deflecting mass of $2.3 \mathrm{~kg}$ loading the $1.0-\mathrm{mm}$-thick top plate. The dashed line refers to calculated frequencies from expressions (4)-(9). Here we note that formulas (4)-(7) calculate resonant frequencies to within a typical error of $5 \%$ in the range of parameters ${ }^{10}$

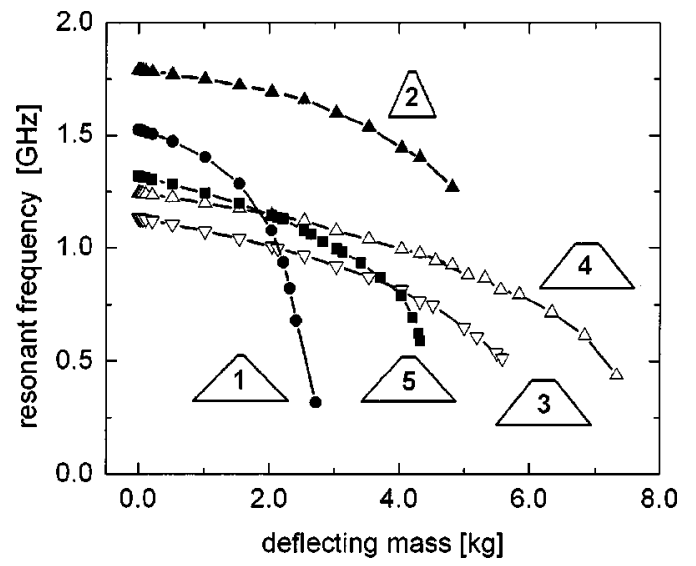

FIG. 8. For several inserts, the dependence of measured frequencies on the loading weight.

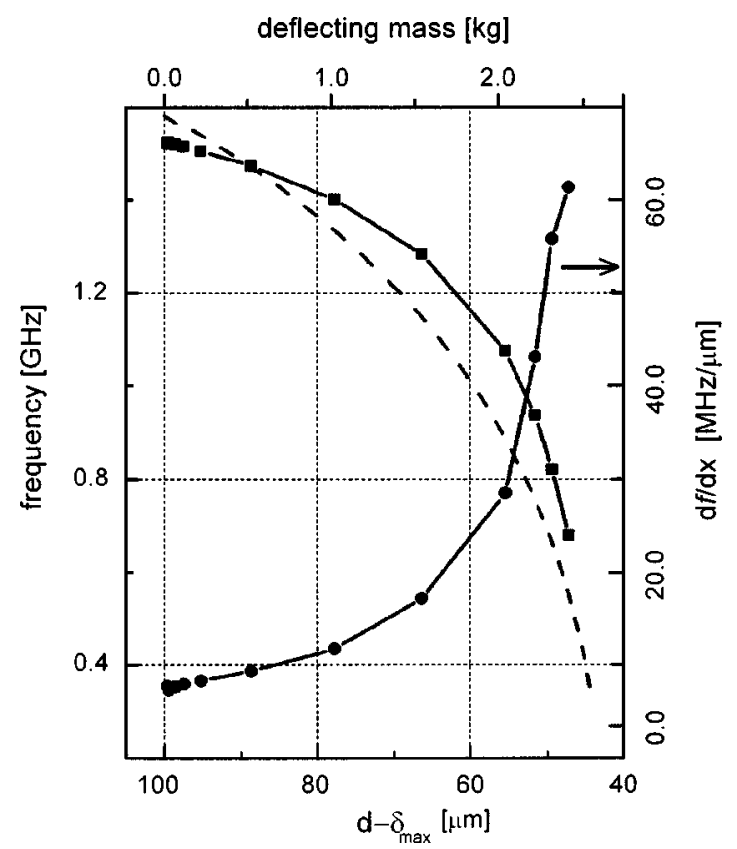

FIG. 9. Resonant frequency and displacement sensitivity $d f / d x$ for insert 1 expressed in terms of the decreasing dynamical gap $x=d_{0}-\delta_{\max }$. The dashed line refers to calculated frequencies.

$$
\begin{aligned}
& r_{0} / l_{M} \geqslant 1 / 3, \\
& \left(r_{2}-r_{0}\right) / l_{M} \geqslant 1 / 3+3 l_{M} / \lambda_{0},
\end{aligned}
$$

where the characteristic length $l_{M}$ is defined in Eq. (7). For the present case referring to insert 1 , relations (10) are marginally satisfied since both $r_{2}-r_{0} / l_{M}=0.65$ and $r_{0} / l_{M}$ $=0.06$ are rather small. Nevertheless, the calculated curve in Fig. 9 fits the measured frequencies within an error of $12 \%$.

\section{DISCUSSION}

We have discussed the feasibility of a $1.0 \mathrm{GHz}$ reentrant cavity as a parametric transducer by demonstrating experimentally the transducer sensitivity to deflections of the 80.0mm-diam, 1.0-mm-thick aluminum plate when loaded with weights as light as $10 \mathrm{~g}$. Through proper selection of the cavity geometry by increasing $r_{1}$ (with $r_{2}$ and $l$ fixed) and reducing both $\bar{r}_{0}$ and the gap $d$, typical tuning coefficients $\Delta f_{0} / \Delta d$ of a few tens of $\mathrm{MHz} / \mu \mathrm{m}$ have been achieved at gap spacings in the 50-100 $\mu \mathrm{m}$ range. In addition to conferring high tuning coefficient, a narrow gap maximizes the electrical coupling to a mechanical transformer that matches the impedance of the transducer to the antenna impedance. ${ }^{12}$ Scaled up by a factor of 10 for ease of machining and testing

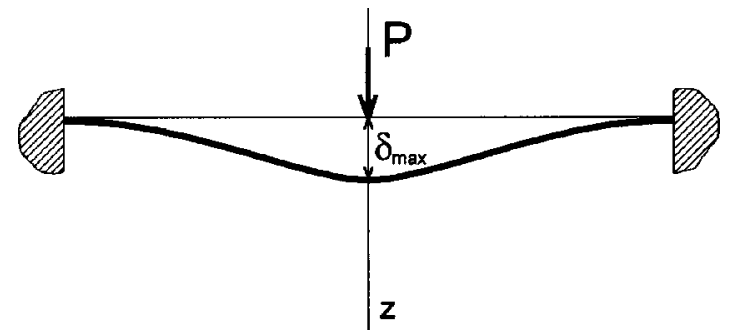

FIG. 10. Deflection of a clamped plate loaded at the center. 
at early stages of the design, the cavities we have examined are developmental replicas of electromechanical transducers to be instrumented in a resonant mass gravitational wave antenna, the Schenberg detector, under development at the National Institute for Space Research. ${ }^{13}$ Specific requirements for the tuning coefficient in this kind of detector is placed by the sources of noise, which may be divided in two groups, ${ }^{14,15}$ namely, narrow- and broad-band noises. Narrowband-noise is characterized by fluctuations due to the back action effect from the pump oscillator and goes up with the square of the sensitivity $\Delta f_{0} / \Delta d$. On the other hand, broadband noise has two components, the serial noise (arising from noise temperature of the microwave amplifier in the readout chain $^{7}$ ) and the phase noise (due to phase fluctuations of the incident pump oscillator). Both of these components fall as the square of $\Delta f_{0} / \Delta d$, and so an optimum value for $\Delta f_{0} / \Delta d$ can be found that balances the narrow-band noise with the broad-band noise. Therefore, the transducer $\Delta f_{0} / \Delta d$ ratio useful for gravitational wave detection application is highly dependent on the electrical quality factor of the microwave cavity and other transducer parameters, e.g., pump oscillator amplitude and phase noise, incident power, and transducer temperature. For typical values of these parameters, $\Delta f_{0} / \Delta d$ lies in the range of $10-1000 \mathrm{MHz} / \mu \mathrm{m}$. As a technical goal for the Schenberg detector, ${ }^{13}$ the strain noise power spectral density is targeted at $2 \times 10^{-23} \mathrm{~Hz}^{-1 / 2}$ to achieve the standard quantum limit, thereby requiring an optimum frequency tuning coefficient of $850 \mathrm{MHz} / \mu \mathrm{m}$ upon including the amplitude and phase noises at the levels of -165 and $-145 \mathrm{dBc} / \mathrm{Hz}$, respectively. In this experiment the cavity-based transducer, which actually operates at 10.0 $\mathrm{GHz}$, is expected to be a hundred times more sensitive (see the Appendix) than the oversized cavities tested here.

\section{ACKNOWLEDGMENTS}

This work has been supported by the National Council for Scientific and Technological Development (CNPq) and the São Paulo State Research Foundation (FAPESP), Brazil.

\section{APPENDIX: SCALING RELATIONSHIPS AND DERIVATION OF AN EXPRESSION FOR THE TUNING COEFFICIENT $\Delta f_{0} / \Delta d$}

To examine scaling relationships, we consider two similar reentrant cavities (Fig. 3) with linear dimensions in the ratio $\beta$, namely,

$$
\beta=\frac{\bar{r}_{0}^{\prime}}{\bar{r}_{0}}=\frac{r_{0}^{\prime}}{r_{0}}=\frac{r_{1}^{\prime}}{r_{1}}=\frac{r_{2}^{\prime}}{r_{2}}=\frac{l^{\prime}}{l}=\frac{d^{\prime}}{d} .
$$

Since the angle $\alpha$ remains the same and the lumped electrical parameters $L$ and $C$ are proportional to length, we have from Eqs. (4)-(8),

$$
\frac{C_{0}^{\prime}}{C_{0}}=\frac{C_{1}^{\prime}}{C_{1}}=\frac{L^{\prime}}{L}=\beta,
$$

and therefore the corresponding resonant frequencies are related by the inverse ratio

$$
\frac{f_{0}^{\prime}}{f_{0}}=\frac{1}{\beta} .
$$

By differentiating $f_{0}$ with respect to the gap spacing $d$ we obtain the following expression for the tuning coefficient:

$$
\begin{aligned}
\frac{\Delta f_{0}}{\Delta d}= & \frac{1}{2} \frac{f_{0}}{d}\left[\frac { 1 } { T _ { 3 } } \left\{\frac{r_{0}^{3}}{d}+\frac{2 r_{0} l \cot ^{2} \alpha}{r_{1}-\bar{r}_{2}}-\left(T_{1}+T_{2}\right) \frac{2 d \cos ^{2} \alpha}{\alpha^{2}\left(r_{1}-\bar{r}_{0}\right)}\right.\right. \\
& -\frac{2 d}{\alpha}\left[\frac{1}{2}\left(\cot \alpha+\frac{d \cot ^{2} \alpha}{r_{1}-\bar{r}_{0}}\right) \ln \frac{\sqrt{e} l_{M} \sin \alpha}{d}\right. \\
& -\left(1+\frac{d \cos ^{2} \alpha}{r_{1}-\bar{r}_{0}} \cot \alpha\right) \frac{\cot \alpha}{2}-\frac{l \cot ^{2} \alpha}{r_{1}-\bar{r}_{0}} \ln \frac{e l_{M} \sin \alpha}{d} \\
& \left.\left.\left.-\frac{r_{0}}{d}\left(1+\frac{d \cos ^{2} \alpha \cot \alpha}{r_{1}-\bar{r}_{0}}\right)+T_{1 X}+T_{2 X}\right]\right\}\right],
\end{aligned}
$$

where

$$
\begin{aligned}
& T_{1}=r_{0} \ln \frac{e l_{M} \sin \alpha}{d}, \\
& T_{2}=\frac{d \cot \alpha}{2} \ln \frac{\sqrt{e} l_{M} \sin \alpha}{d}, \\
& T_{3}=\frac{r_{0}^{2}}{d}+\frac{2}{\alpha}\left(T_{1}+T_{2}\right), \\
& T_{1 X}=\frac{r_{0}}{l_{M}} \partial_{d} l_{M}\left[r_{0}(d)\right], \\
& T_{2 X}=\frac{d \cot \alpha}{2 l_{M}} \partial_{d} l_{M}\left[r_{0}(d)\right] .
\end{aligned}
$$

Because $T_{3}$ and each of the terms inside the brackets is proportional to length, i.e., the overall braced term is dimensionless, it follows that the tuning coefficient scales as the square of the inverse ratio:

$$
\left(\frac{\Delta f_{0}^{\prime}}{\Delta d^{\prime}}\right) /\left(\frac{\Delta f_{0}}{\Delta d}\right)=\frac{1}{\beta^{2}} .
$$

Thus, if a low-frequency cavity is scaled down by a factor of 10 , i.e., all the dimensions are multiplied by $\beta=1 /$ 10 , the resulting tuning coefficient for the high-frequency cavity becomes 100 times as high as in the larger, lowerfrequency cavity.

In addition, expression (A1) for $\Delta f_{0} / \Delta d$ shows that the sensitivity depends only on the internal dimensions of the cavity, being independent of the thickness of the top plate. While maintaining the internal dimensions constant, reduction of the plate thickness, for example, from 1.0 to $0.464 \mathrm{~mm}$ does not imply a tenfold increase in the sensitivity. By noting that $\Delta f_{0} / \Delta d=(\Delta P / \Delta d)\left(\Delta f_{0} / \Delta P\right)$, in this case the sensitivity $\Delta f_{0} / \Delta d$ is not improved, but instead the ratio of change of applied force with displacement, $\Delta P / \Delta d$, is the quantity that becomes ten times larger. In fact, since $h=(0.464)^{3} \mathrm{~mm} \cong 1.0 \mathrm{~mm} / 10$ and according to Eq. (9), we readily arrive at $\left.\left(P / \delta_{\max }\right)\right|_{h=0.464 \mathrm{~mm}}=(1 / 10)$ $\times\left.\left(P / \delta_{\max }\right)\right|_{h=1.0 \mathrm{~mm}}$. 
${ }^{1}$ J. C. Slater, Microwave Electronics (Dover, New York, 1969), pp. 232 237.

${ }^{2}$ S. Humphries, Jr., Principles of Charged Particle Acceleration (Wiley, New York, 1986).

${ }^{3}$ P. Kneisel, in Proceedings of the 8th European Particle Accelerator Conference (European Physical Society, Inter-divisional Group on Accelerators, Geneve, 2000), pp. 139-143.

${ }^{4}$ V. A. Dolgashev, in Proceedings of the XXI International LINAC Conference (Stanford Linear Accelerator Center, Stanford, CA, 2002), pp. 266270.

${ }^{5}$ T. M. Antonsen, Jr., A. A. Mondell, B. Levush, J. P. Verboncoeur, and C. K. Birdsall, Proc. IEEE 87, 804 (1999).

${ }^{6}$ Computer Simulation Technology GmbH, computer code CST Microwave Studio (Darmstadt, Germany, 1998).

${ }^{7}$ D. G. Blair, E. N. Ivanov, M. E. Tobar, P. J. Turner, F. van Kann, and I. S. Heng, Phys. Rev. Lett. 74, 1908 (1995).

${ }^{8}$ P. J. Veitch, D. G. Blair, N. P. Linthorne, L. D. Mann, and D. K. Ramm,
Rev. Sci. Instrum. 58, 1910 (1987).

${ }^{9}$ N. P. Linthorne and D. G. Blair, Rev. Sci. Instrum. 63, 4154 (1992).

${ }^{10}$ K. Fujisawa, IRE Trans. Microwave Theory Tech. 6, 344 (1958).

${ }^{11} \mathrm{~S}$. Timoshenko and S. Woinowsky-Krieger, Theory of Plates and Shells (McGraw-Hill, New York, 1959), p. 69

${ }^{12}$ P. J. Turner, Ph.D. thesis, University of Western Australia, 1995.

${ }^{13}$ O. D. Aguiar, L. A. Andrade, J. J. Barroso, L. Camargo Filho, L. A. Carneiro, C. S. Castro, P. J. Castro, C. A. Costa, K. M. F. Costa, J. C. N. de Araujo, A. U. de Lucena, W. de Paula, E. C. de Rey Neto, S. T. de Souza, A. C. Fauth, C. Frajuca, G. Frossati, S. R. Furtado, L. C. Lima, N. S. Magalhães, R. M. Marinho Jr., E. S. Matos, J. L. Melo, O. D. Miranda, N. F. Oliveira Jr., B. W. Paleo, M. Remy, K. L. Ribeiro, C. Stellati, W. F. Velloso, and J. Weber, Class. Quantum Grav. 21, S457 (2004).

${ }^{14}$ M. E. Tobar, C. R. Locke, E. N. Ivanov, I. S. Heng, and D. G. Blair, Rev. Sci. Instrum. 71, 4282 (2000).

${ }^{15}$ M. E. Tobar, Physica B 280, 520 (2000). 